\title{
Endobronchial metastasis: An epidemiologic and clinicopathologic study of 174 consecutive cases
}

\author{
Alessandro Marchioni ${ }^{a, *}$, Anna Lasagni ${ }^{a}$, Annalisa Busca ${ }^{b}$, Alberto Cavazza $^{c}$, \\ Lorenzo Agostini $^{\mathrm{d}}$, Mario Migaldi ${ }^{\mathrm{e}}$, Paolo Corradini ${ }^{\mathrm{a}}$, Giulio Rossi ${ }^{\mathrm{e}}$ \\ a Department of Oncology and Hematology, Respiratory Diseases Clinic, Azienda Ospedaliero-Universitaria Policlinico, Modena, Italy \\ b Operative Unit of Pulmonology, Hospital "Cattinara", Trieste, Italy \\ c Operative Unit of Pathologic Anatomy, Azienda Ospedaliera Arcispedale S. Maria Nuova-IRCCS, Reggio Emilia, Italy \\ d Operative Unit of Pulmonology, Azienda Ospedaliera Arcispedale S. Maria Nuova-IRCCS, Reggio Emilia, Italy \\ e Department of Diagnostic Laboratories, Operative Unit of Pathologic Anatomy, Azienda Ospedaliero-Universitaria Policlinico, Modena, Italy
}

\section{A R T I C L E I N F O}

\section{Article history:}

Received 10 January 2014

Received in revised form 23 February 2014

Accepted 2 March 2014

\section{Keywords:}

Lung

Metastasis

Bronchus

Immunohistochemistry

Endobronchial

Bronchoscopy

\begin{abstract}
A B S T R A C T
Purpose: Endobronchial metastases from extrapulmonary solid tumors are a rare event and currently available epidemiological and clinico-pathological data mainly derive from anecdotal case reports. Methods: A series of 174 consecutive cases of endobronchial metastases from extrathoracic solid tumors were collected over a period of 18 years. Immunohistochemistry was performed in 115 cases. Complete imaging features were available in 81 patients, and analysis of the latency period between primitive tumor diagnosis and occurrence of endobronchial metastasis was obtained.

Results: Among all bronchoscopic examinations performed in the same period for malignancy, a mean of 5.6 cases per year consisted of endobronchial metastases (range 2-17 cases), with a statistically significant increase when comparing the periods 1992-2000 (65 cases, 37\%) and 2001-2009 (109 cases, 63\%) $(p=0.05)$. Overall, $4 \%$ of endobronchial biopsies for suspected malignancy disclosed an endobronchial metastasis from extrapulmonary tumor. Breast ( 52 cases, 30\%), colorectal (42 cases, $24 \%$ ), renal (14\%), gastric (6\%) and prostate (4.5\%) cancers and melanoma (4.5\%) were the most common metastatic neoplasms presenting as endobronchial mass. One-hundred fifty-four cases were identified after the primitive tumor diagnosis (metachronous cases, 89\%), 11 cases were simultaneously evidenced in extrapulmonary and endobronchial sites (synchronous cases, 6\%), while 9 occult metastatic cases (5\%) first presented as endobronchial mass (anachronous cases). Overall, mean latency from extrapulmonary tumor diagnosis and endobronchial metastasis was 136 months (range, 1-300 months). The most frequent symptoms were dyspnea $(23 \%)$, cough $(15 \%)$ and haemoptysis $(12 \%)$, while $26 \%$ of patients were totally asymptomatic. At radiology, 53\% presented as multiple pulmonary nodules, while other cases presented as hilar and mediastinal mass, single peripheral nodule, atelectasis or pleural effusion.

Conclusions: Endobronchial metastases from extrapulmonary tumors account for about $4 \%$ of all bronchoscopic biopsies performed for suspected malignancy and in $5 \%$ of the cases the metastasis is the first manifestation of the neoplasm.
\end{abstract}

(c) 2014 Elsevier Ireland Ltd. All rights reserved.

\section{Introduction}

Metastatic disease to the lungs is a common occurrence in routine oncologic practice and $20-50 \%$ of primary extrapulmonary solid malignancies show pulmonary metastases during their

\footnotetext{
* Corresponding author at: Respiratory Diseases Clinic, Azienda OspedalieroUniversitaria Policlinico, via del Pozzo, 71, 41124 Modena, Italy. Tel.: +39 059 4225859; fax: +390594222571.

E-mail address: marchioni.alessandro@unimore.it (A. Marchioni).
}

biologic course [1,2]. Among all clinico-radiologic presentations, the finding of metastatic tumors manifesting as endobronchial masses is a rare and possibly underestimated event.

Epidemiological studies show a broadly variable incidence of endobronchial metastases ranging from 2 to $50 \%$ depending on several factors, including the different clinical settings and/or the length of the period of time considered, the ethnic background which is significantly associated with different incidences of tumor types, as well as the criteria used to identify this unusual occurrence [3-6]. Kiryu et al. [7] proposed a 4 types classification of the development pattern of endobronchial metastases, as follows: type 
I: direct metastasis to the bronchus; type II: bronchial invasion by parenchymal lesion; type III: bronchial invasion by mediastinal or hilar lymph node metastasis; type IV: peripheral lesion extending along the proximal bronchus. However, the great majority of endobronchial metastases reported in the literature are of type I due to the difficulty to discriminate these 4 patterns with certainty.

On the clinical ground, it is noteworthy that several patients may be totally asymptomatic and radiological findings are not particularly helpful in hindering differential diagnosis between endobronchial primary and metastatic tumors [6].

Among solid tumors occurring as endobronchial metastases, carcinomas from breast, kidney and colon-rectum are the most commonly encountered [4,7-30]. The timeframe between primitive tumor diagnosis and appearance of endobronchial metastases is quite variable although usually long, with an average of 50 months [7-9]. Finally, endobronchial metastases may generally follow identification of the primary site (metachronous cases) or more rarely precede (anachronous) or simultaneously reveal (synchronous) the primary malignancy discovery. Currently, our knowledge on endobronchial metastases from extrapulmonary solid tumors derive from case reports and few studies with a limited series of cases or conducted by using different clinical and radiological data collection methods, then resulting in fragmented and/or controversial data.

The aim of our study is to draw a more homogeneous epidemiological, clinico-radiologic and pathologic scenario of endobronchial metastases through a careful retrospective analysis of 174 consecutive cases along a period of 18 years collected from 2 different Institutions. The results herein presented were then compared with experiences previously appeared in the literature, in the hope to identify helpful features for suspecting metastatic disease when dealing with endobronchial masses.

\section{Materials and methods}

A series of 174 consecutive cases of endobronchial metastasis from extrapulmonary tumors were collected from the Units of Pathologic Anatomy and Pulmonology of the Azienda OspedalieroUniversitaria Policlinico of Modena and the Azienda Ospedaliera St. Maria Nuova of Reggio Emilia. Diagnoses were made between January 1992 and December 2009 and all cases were first independently diagnosed and then reviewed at a multiheaded microscope by two expert pulmonary pathologists (AC, GR). Endobronchial metastasis was recorded when a bronchoscopically visible, extrapulmonary neoplasm metastasized to the central or segmental bronchus. Lymphoproliferative malignancies were excluded from the study, since lymphomas or plasma cell neoplasms may frequently arise from the mediastinum or involve lymph nodes of this anatomic site then directly invading the bronchial structures. All cases consisted of bronchial biopsies that were formalinfixed and paraffin-embedded. In all cases, 4-micron sections from the paraffin-block were performed for routine staining with hematoxylin-eosin. However, in 115 cases (66\%) the diagnosis required further analysis by means of immunohistochemical stains. When required, immunohistochemistry was performed using an automated immunostainer (Benchmark, Ventana, Tucson, AZ). Both institutions independently performed immunohistochemical analysis, but using the same type of instruments as well as the same antibody clones. The panel of antibodies in each single case was appropriately selected depending on the differential diagnosis. The primary antibodies used in the study were the following: Thyroid Transcription Factor-1/TTF-1 (clone 8G7G3/3, Ventana, prediluted), Thyroglobulin (clone $2 \mathrm{H} 11 / 6 \mathrm{E} 1$, Ventana, prediluted), CDX2 (clone EPR2764Y, Ventana, prediluted), CD10 (clone 013, Ventana, prediluted), estrogen receptors (clone SP1, Ventana; prediluted), progesterone receptors (clone 1E2, Ventana, prediluted), Wilms'Tumor-1/WT-1 (clone 6F-H2, Ventana, prediluted), calretinin (clone SP65, Ventana, prediluted), S100 (polyclona, Ventana, prediluted), melan-A (clone MART-1, Ventana, prediluted), PSA (ER PR8, Ventana, prediluted), smooth-muscle-actin (clone $1 \mathrm{~A} 4$, Ventana, prediluted), desmin (clone DE-R-11, Ventana, prediluted), pan-cytokeratins (clone AE1/AE3, Ventana, prediluted), CD34 (clone QB-END/10, Ventana, prediluted), CD31 (clone JC/70A, Ventana, prediluted) (6F-H2, Ventana, preduted), p63 (clone 4A4, Ventana, prediluted), calcitonin (polyclonal, Ventana, prediluted), Epithelial Membrane Antigen/EMA (clone E29, Ventana, prediluted).

Clinical data (sex, age, symptoms, time lapse between primary and metastatic tumors, type of primary extrapulmonary tumor) were available in all cases, while detailed imaging features were obtained in 81 cases.

\subsection{Statistical analysis}

Contingency tables were used for descriptive and comparative statistical analysis of collected data, and significance was evaluated with Pearson's chi-squared test and Fisher's test. All statistical calculations were performed using SPSS 13.0 software (Statistical Package for the Social Sciences, Chicago, IL). Differences were considered statistically significant for probability $<0.05$.

The study was conducted in accordance with the precepts of the Helsinki Declaration and all recorded data were handled anonymously.

\section{Results}

The baseline characteristics of the entire case series are summarized in Table 1.

The case histories collected included 174 cases of endobronchial metastasis, with a slight prevalence of male patients (54\%), an average age of 67 years and a range between 27 and 89 years. Diagnosis of endobronchial metastasis was obtained after a diagnosis of extrapulmonary tumor (metachronous cases) in 154 cases (89\%), while in 11 cases $(6 \%)$ the tumor was detected on an endobronchial level simultaneously to that on the extrapulmonary site (synchronous cases). In the remaining 9 cases (5\%), the endobronchial metastasis was the first manifestation of an occult extrapulmonary tumor (anachronous cases). Distribution of the primary tumors and time of endobronchial metastasis occurrence is summarized in Table 2.

It should be noticed that around $50 \%$ of anachronous cases were secondary to renal carcinoma. On average, in the overall 18-year period, 5.6 cases of endobronchial metastasis were observed per year (range: $2-17$ cases), with statistically significant differences for the periods between 1992-2000 (65 cases, 37\%) and 2001-2009 (109 cases, 63\%) $(p=0.05)$.

To allow a better understanding on the extent of the issue and on how this could influence the clinician's activity, we referred to the bronchoscopy service of the Azienda Ospedaliero-Universitaria Policlinico in Modena to review all the reports of the bronchoscopic investigations performed between 2006 and 2009. In these 4 years, 4208 procedures were performed, 781 (18.5\%) on patients for whom endoscopic findings were suggestive of tumors in the tracheobronchial tree. Of note, 31 out of 781 (4\%) broncoscopic examinations revealed endobronchial metastases from nonthoracic malignancies.

Bronchoscopy was performed for the following reasons: radiological features (nodules, masses and hilar-mediastinal and/or peripheral lymphadenomegaly), atelectasis, and symptoms such as dyspnea, haemoptysis and persistent cough. Biopsies were 
Table 1

Clinico-pathologic characteristics of patients with endobronchial metastasis.

\begin{tabular}{|c|c|}
\hline Variable & Frequency (\%) \\
\hline \multicolumn{2}{|l|}{ Sex and age } \\
\hline Male (mean age: 69 years) & $94(54)$ \\
\hline Female (mean age: 66 years) & $80(46)$ \\
\hline \multicolumn{2}{|l|}{ Symptoms } \\
\hline Asymptomatic & $42(24)$ \\
\hline Cough & $38(22)$ \\
\hline Dyspnea & $30(17)$ \\
\hline Haemoptysis & $20(12)$ \\
\hline Dysphonia & $2(1)$ \\
\hline Not available & $24(14)$ \\
\hline \multicolumn{2}{|l|}{ Primary tumors } \\
\hline Breast & $52(30)$ \\
\hline Colon-rectum & $42(24)$ \\
\hline Kidney & $24(14)$ \\
\hline Stomach & $11(6)$ \\
\hline Prostate & $8(4.5)$ \\
\hline Melanoma & $8(4.5)$ \\
\hline Thyroid & $5^{\mathrm{a}}(3)$ \\
\hline Endometrium & $3(2)$ \\
\hline Liver & $3(2)$ \\
\hline Small bowel & $2(1)$ \\
\hline Ovary & $2(1)$ \\
\hline Leiomyosarcoma & $3^{\mathrm{b}}(2)$ \\
\hline Bladder & $2(1)$ \\
\hline Renal pelvis & $1(0.5)$ \\
\hline Mesothelioma & $1(0.5)$ \\
\hline Solitary fibrous tumor & $1(0.5)$ \\
\hline Vagina & $1(0.5)$ \\
\hline Cervix & $1(0.5)$ \\
\hline Esophagus & $1(0.5)$ \\
\hline Liposarcoma spermatic cord & $1(0.5)$ \\
\hline Nasopharynx & $1(0.5)$ \\
\hline Meningioma & $1(0.5)$ \\
\hline \multicolumn{2}{|l|}{ Type of metastasis } \\
\hline Metachronous & $154(89)$ \\
\hline Synchronous & $11(6)$ \\
\hline Anachronous & $9(5)$ \\
\hline
\end{tabular}

a 3 papillary type, 1 each anaplastic and medullary type.

b 2 cutaneous type and 1 uterine type.

Table 2

Distribution of metastasis primary and time of endobronchial metastasis occurrence.

\begin{tabular}{|c|c|c|c|}
\hline Primary tumors & $\begin{array}{l}\text { Metachronous } \\
154(89 \%)\end{array}$ & $\begin{array}{l}\text { Synchronous } \\
11(6 \%)\end{array}$ & $\begin{array}{l}\text { Anachronous } \\
9(5 \%)\end{array}$ \\
\hline Breast $=52$ & 48 & 2 & 2 \\
\hline Colon-rectum $=42$ & 40 & 2 & - \\
\hline Kidney $=24$ & 19 & 1 & 4 \\
\hline Stomach $=11$ & 6 & 4 & 1 \\
\hline Prostate $=8$ & 7 & - & 1 \\
\hline Melanoma $=8$ & 8 & - & - \\
\hline Thyroid $=5^{\mathrm{a}}$ & 5 & - & - \\
\hline Endometrium $=3$ & 3 & - & - \\
\hline Liver $=3$ & 3 & - & - \\
\hline Small bowel $=2$ & 2 & - & - \\
\hline Ovary $=2$ & 2 & - & - \\
\hline Leiomyosarcoma $=3^{b}$ & 3 & - & - \\
\hline Bladder $=2$ & 1 & 1 & - \\
\hline Renal pelvis $=1$ & - & - & 1 \\
\hline Mesothelioma = 1 & 1 & - & - \\
\hline Solitary fibrous tumor $=1$ & 1 & - & - \\
\hline Vagina $=1$ & 1 & - & - \\
\hline Cervix $=1$ & 1 & - & - \\
\hline Esophagus $=1$ & - & 1 & - \\
\hline Liposarcoma spermatic cord $=1$ & 1 & - & - \\
\hline Nasopharynx $=1$ & 1 & - & - \\
\hline Meningioma $=1$ & 1 & - & - \\
\hline
\end{tabular}

a 3 papillary type, 1 each anaplastic and medullary type.

b 2 cutaneous type and 1 uterine type. performed on macroscopically visible endobronchial lesions without complementary methods.

On the full cohort of 174 patients, extrapulmonary tumors most often resulting in endobronchial metastasis included breast carcinoma (52 cases, 30\%), colorectal carcinoma (42 cases, $24 \%$ ), renal carcinoma ( 24 cases, $14 \%$ ), stomach carcinoma (11 cases, $6 \%$ ), prostate carcinoma ( 9 cases, $5 \%$ ), and melanoma ( 8 cases, $4.5 \%$ ).

Although endobronchial metastases from epithelial-derived tumors (carcinomas) significantly overridden mesenchymal neoplasms ( 5 cases) and melanomas ( 8 cases), it is important to notice that virtually all types of solid tumor can metastasize into the bronchial tree. Supporting this, three types of tumor included in the current series (liposarcoma of the spermatic cord, solitary fibrous tumor and cutaneous leiomyosarcoma) have not so far reported in the literature (Figs. 1 and 2).

Although not statistically significant, endobronchial metastases more frequently affected the right (102 cases, 59\%) than the left bronchus ( 72 cases, $41 \%)(p=0.07)$.

The overall median latency period between the detection of extrapulmonary tumor and the occurrence of endobronchial metastasis was 136 months (range, 1-300 months). Among the most frequent extrapulmonary malignancies leading to endobronchial metastasis, a significant difference was observed between breast carcinoma (median, 86 months; range, 1-300 months) or renal carcinoma (median, 82 months; range, 18-270 months) and colorectal carcinoma (median, 53 months; range, 9-168 months) $(p<0.001)$ (Fig. 3).

\subsection{Clinico-radiologic features}

The patterns of clinical and radiological presentation, ordered by frequency, for the 81 analyzed patients were summarized in Table 3 (supplement material) and Table 4 (supplement material), respectively. Of the collected case histories, 21 patients (26\%) were asymptomatic, while the most common symptoms reported were dyspnea, cough and haemoptysis, in 19 (23\%), 15 (19\%) and 10 $(12 \%)$ cases respectively. In one single case the only symptom was dysphonia secondary to left laryngeal nerve compression. For 16 patients, the presenting symptoms were not reported in clinical charts.

See Tables 3 and 4 as supplementary files. Supplementary material related to this article can be found, in the online version, at http://dx.doi.org/10.1016/j.lungcan.2014.03.005.

Only standard chest x-rays were available for 7 patients, while chest $\mathrm{x}$-rays and CT scans were available for the remaining patients. "Nodules" were defined as lesions with a maximum diameter equal to or less than $30 \mathrm{~mm}$; "masses" were defined as lesions with a diameter over $30 \mathrm{~mm}$; "lymphadenomegaly" was defined as hilar-mediastinal lymph nodes with a maximum diameter over $10 \mathrm{~mm}$. Multiple nodules were present in 43 cases (53\%), hilar-mediastinal lymphadenomegaly in 38 cases (47\%), a peripheral mass in 24 cases (30\%), atelectasis in 23 cases (28\%), pleural effusion in 19 cases (23\%) and a hilar-mediastinal mass in 13 cases (16\%).

\section{Discussion}

Lungs are the commonest site of metastatic deposits from extra-pulmonary malignancies, but metastases presenting as endobronchial growth are quite unusual and epidemiologic and/or clinic-pathologic data on large homogeneous series are lacking. The present study collected the largest series to date of consecutive endobronchial metastases from extrapulmonary tumors disclosed in routine practice without selection biases (174 cases), underlying the clinically relevant occurrence of this phenomenon. 

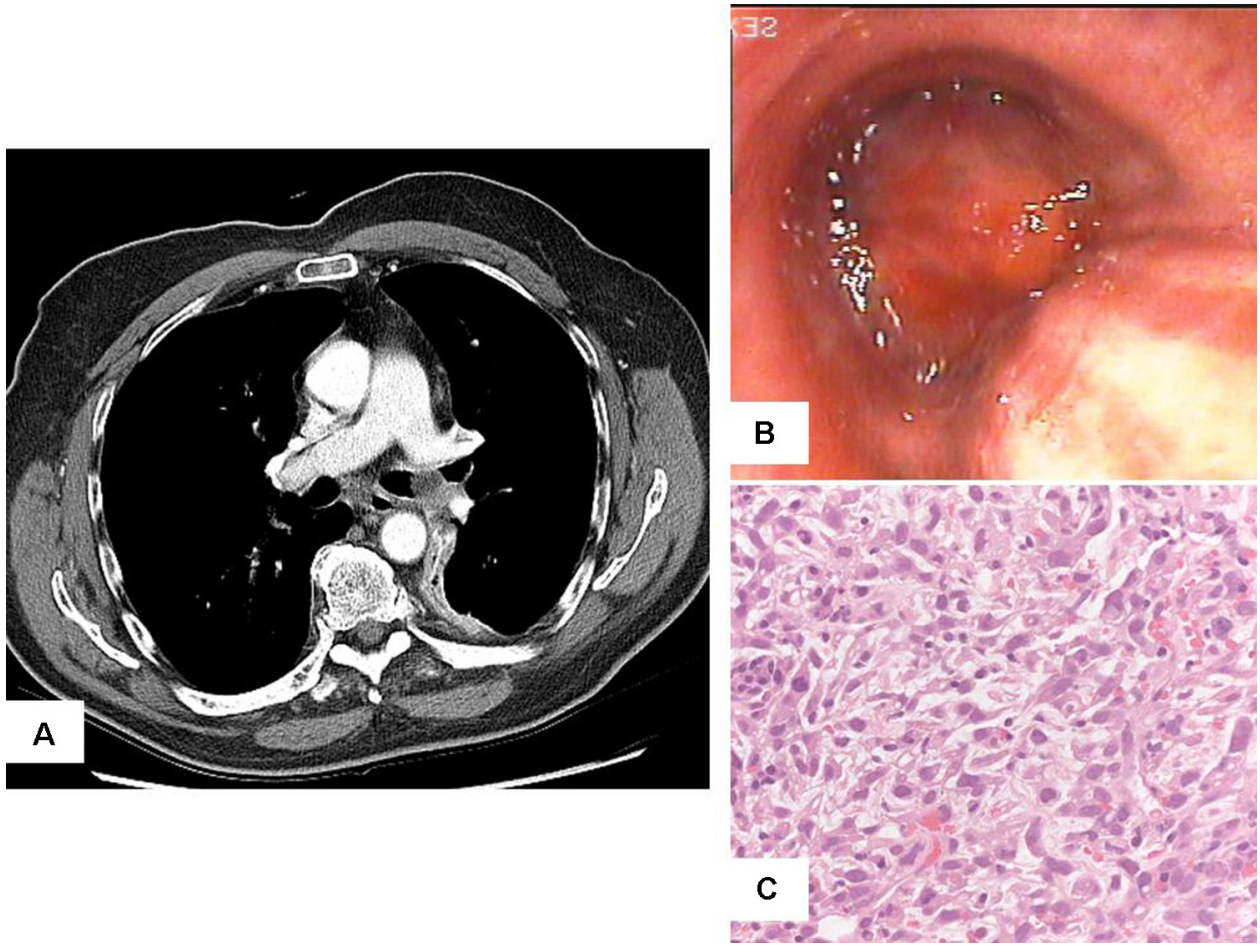

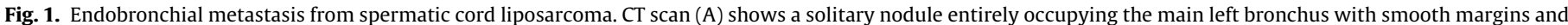
yellowish appearance at bronchoscopy (B). Histology (C) reveals a haphazard proliferation of atypical spindled-shaped cells with clear cytoplasm.

Among all bronchoscopic procedures performed in the suspicion of lung tumor, the overall incidence was about $4 \%$ per year, a figure higher than $1 \%$ reported by Kreisman et al. in 1983 [31]. However, this result is difficult to compare with epidemiological references of other studies, due to the variability of classification criteria in considering endobronchial metastasis, the length and type of the period of time considered as well as the different ethnical background of the study including malignancies with different incidence. It could be reasonable to assume that the increasing number of diagnostic procedures, including bronchoscopy examinations, performed in the current work-up of oncologic patients has lead to higher possibility to disclose endobronchial metastases.
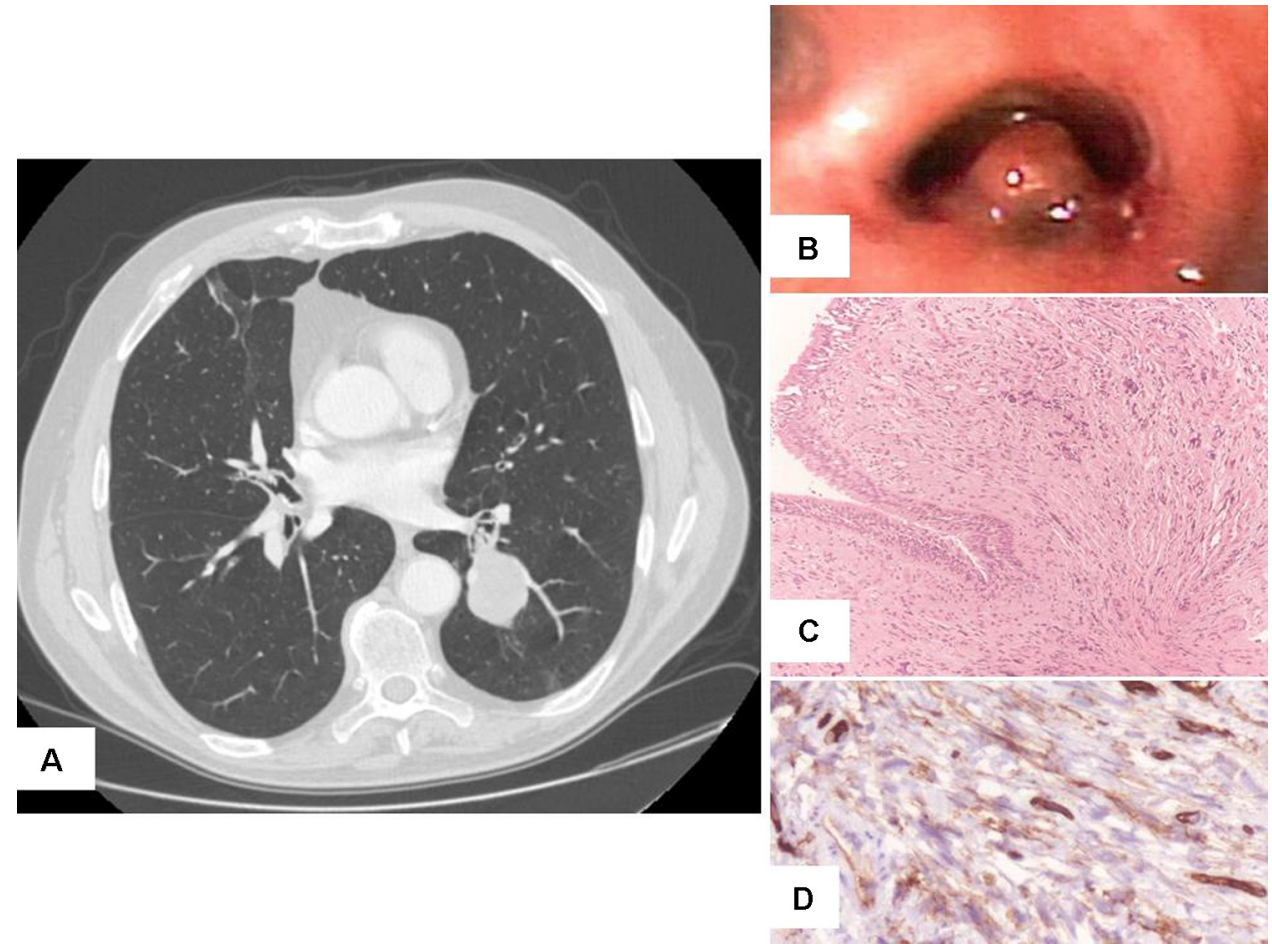

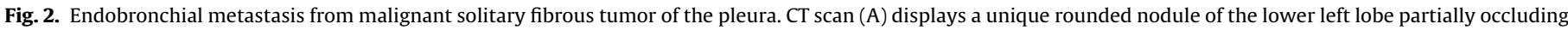
a segmental bronchus (B). Histology shows a submucosal spindle cell proliferation (C) expressing CD34 (D) at immunohistochemistry. 


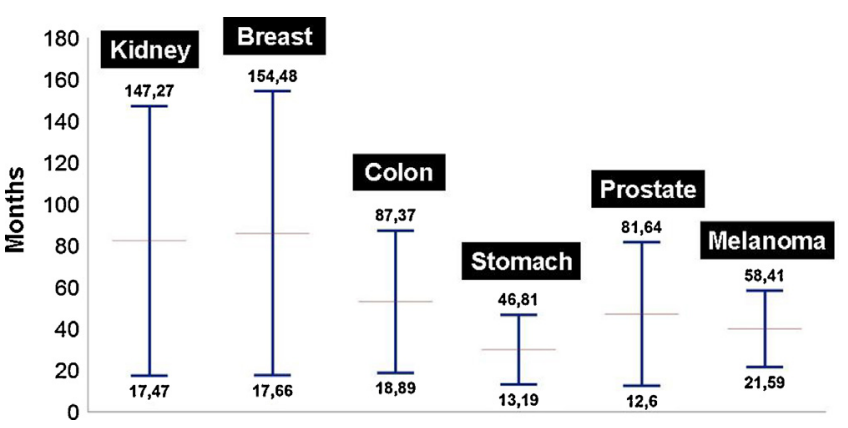

Fig. 3. Distribution of the temporal latency between the occurrence of endobronchial metastases and primary tumors among the most represented malignancies.

In literature, endobronchial metastases generally were reported as single case descriptions or very limited case series $[4,8,32,33]$. Although the development of pulmonary metastases is in fact a common occurrence, their endobronchial location is considered to be a rare event. Frequency estimates are still based on rather outdated studies and primarily on autopsy series [3,6], but it can vary considerably depending on the study and the definition of endobronchial metastasis, ranging from 2 to $50 \%[3,4,12-16,32,33]$. Therefore, the epidemiology of this type of metastasis is still quite unclear.

In our bronchoscopy service, endobronchial metastasis was diagnosed in $4 \%$ of the bioptic procedures performed as a result of suspected malignancy. Considering that bronchoscopy is not routinely performed on all patients with tumors, it may be implied that both incidence and frequency of endobronchial metastases are probably underestimated.

The latency period between primary extrapulmonary tumor and endobronchial metastasis may be very long [36].

For patients presenting with tumors that most commonly cause endobronchial metastasis, the systematic use of chest CT scans and bronchoscopy could be useful. In particular, breast, colon and renal lesions are reported in the literature as being most often associated with endobronchial metastasis [17-24,32,33]. This finding was confirmed in our study that reported how tumors arising in these sites account for over $60 \%$ on the total (see Table 1). For gastric cancer, prostate carcinoma and melanoma, accounting for $16 \%$ of endobronchial metastases in our case series, careful monitoring of the tracheobronchial tract could also facilitate the detection of secondary lesions in a greater number of cases.

Another interesting aspect concerns the capacity of virtually all solid tumors of various cell differentiation to produce endobronchial metastases. So far, this fact was only documented by sporadic single case descriptions [25-30]. Regarding the clinical/radiological presentation, collected data strongly confirm the literature findings [3-6,10,11,32,33], and reassert evidence of a presentation that is difficult to differentiate from that of a primary pulmonary tumor. Among 81 patients for which complete clinico-radiologic data were available, 21 (26\%) were entirely asymptomatic. Previous studies, depending on the case histories included, reported a highly variable percentage of asymptomatic patients, ranging from 0 to $52 \%$ of cases $[8-10,14,32,33]$. When present, dyspnea, cough and haemoptysis were the most common symptoms. Radiological findings evidenced multiple pulmonary nodules and hilar-mediastinal lymphadenomegaly as the most frequent conditions, detected in about half of the examined cases. A peripheral mass and atelectasis were reported in about $1 / 3$ of cases, while pleural effusion and hilar-mediastinal masses were less common.

In some cases, radiological differential analysis vs primitive tumor was extremely difficult, as showed in Fig. 4 where radiological appearance strikingly suggested a primary lung cancer presenting as an excavated pulmonary mass, but the patient had an unknown renal cell carcinoma. In most cases, a diagnosis of endobronchial metastasis follows the diagnosis of the primary lesion, with a largely variable time interval between them. Our data evidenced an average interval of 134 months, with a very

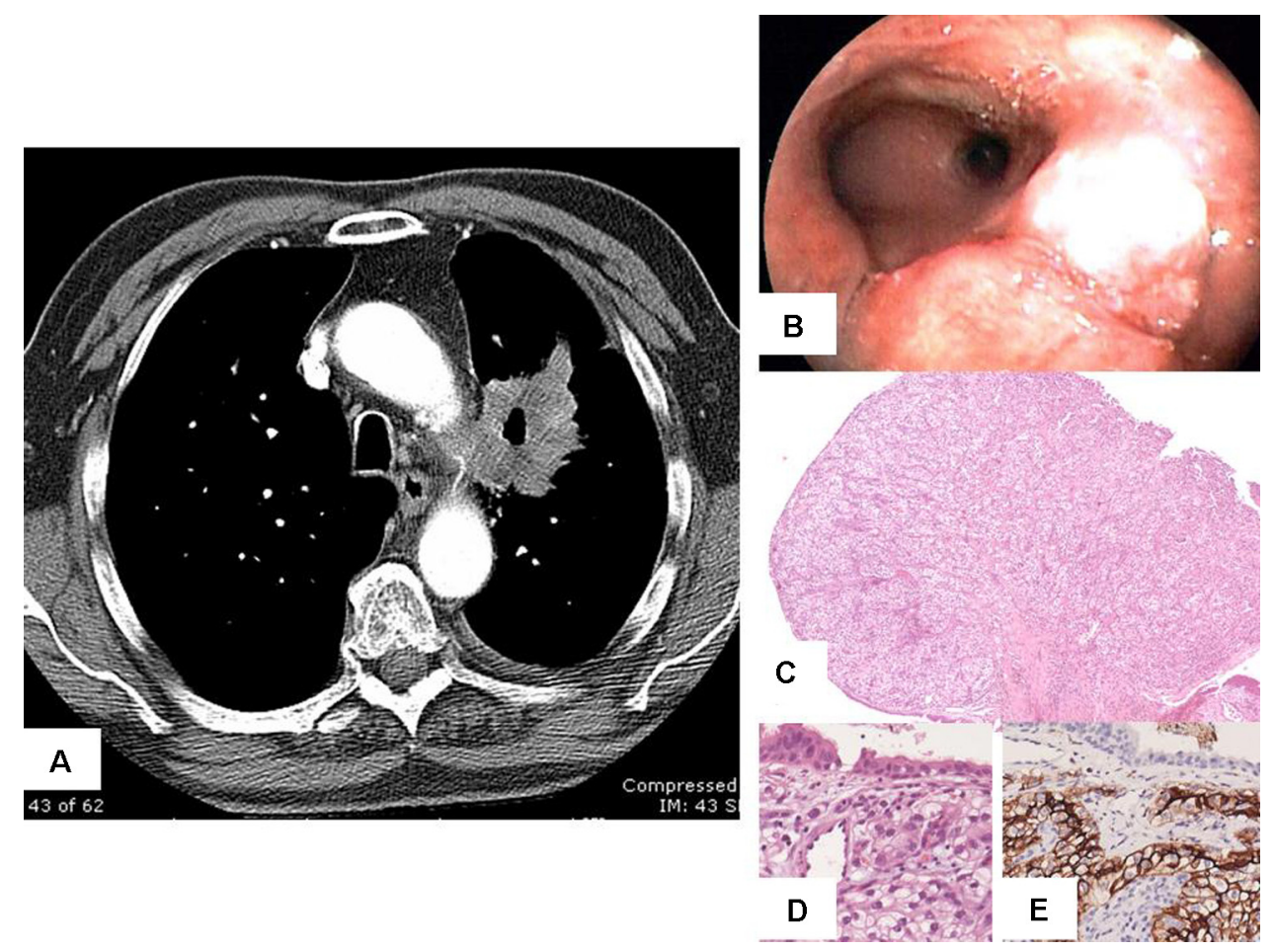

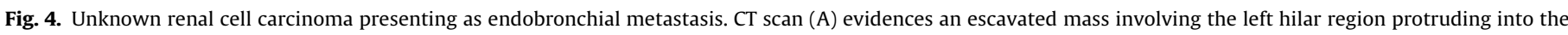
bronchus (B) as an ulcerated polypoid lesion (C) consisting of a submucosal clear cells proliferation (D) expressing CD10 at immunohistochemistry (E). 
wide range accounting up to 300 months, this finding appearing to be significantly discordant when compared to similar observation in previous studies. Soresen, in a review of already published cases, reported an interval of 50 months (range 0-300) [9]; Sebahat evidenced a value of 32.8 months (range 0-96) [10]; Kiryu reported an average interval of 65.3 months [7]; Dursun found a value of 47 months (range $0-19$ years or $0-228$ months) [11]. More recently, Lee et al. [32] evidenced a mean interval of 36 months in a case series of 43 patients, while Kim et al. [33] found a mean interval of 14 months (range, 0-112 months) in their experience on 18 patients. All these intervals are quite shorter than that observed in our study and possibly influenced by many factors, among which the larger number of patients and the greater spectrum of histologic types present in our study. Although metastasis was detected following the diagnosis of the primary lesion in the great majority of our cases, the finding of $5 \%$ of cases in which the primary lesion was unknown when endobronchial biopsy was performed does not appear a negligible feature. Four of these anachronous 9 cases were renal cell carcinomas, while the others consisted of 2 breast cancers, and 1 case each of carcinoma from stomach, prostate and upper urothelial tract (renal pelvis).

Similarly to our results, a previous study on endobronchial metastases secondary to renal carcinoma evidenced that in 7 out of 17 patients the endobronchial metastases occurred before the diagnosis of renal carcinoma [23].

Considering the high incidence and long survival, it is not surprising that, in agreement with previous works on endobronchial metastases, breast and colon cancers are the most commonly described extrapulmonary malignancies showing this peculiar endobronchial growth pattern. However, along a period of 18 years, we found 174 endobronchial metastases disclosing 22 different histologic types, including also tumor entities previously unreported, such as a spermatic cord liposarcoma, a recurrent malignant solitary fibrous tumor, 3 leiomyosarcomas ( 2 from the skin and 1 from the uterus) and a meningioma. Pathologists should then be aware of the occurrence of endobronchial metastases when dealing with a neoplasm showing an unusual morphology for a primary lung tumor and clinico-radiologic data discrepantly supporting a pulmonary malignancy (Figs. 1, 2 and 4).

Unfortunately, the perfect panel of immunistains in detecting primary vs metastatic tumors or in disclosing the type of metastatic neoplasms does not exist. The correct diagnosis always requires a multidisciplinary approach and should be contextualized in each single case based on clinical data, imaging studies, careful morphologic examination and appropriate immunohistochemical markers $[34,35]$. In this study immunomarkers were asked in 115 cases (66\%), but pathologists, as well as clinicians, should be aware that none of the antibodies here employed is absolutely diagnostic per se. Supporting this fact, the metastatic meningioma was originally misdiagnosed as squamous cell carcinoma based on imaging studies revealing a single centrally-located nodule, p63 expression and lack of TTF-1 reactivity. However, the knowledge of a previous diagnosis of recurrent cerebral meningioma has lead to further subtyping tumor cells finally disclosing progesterone receptors and EMA expression, then leading to the correct diagnosis of endobronchial metastasis from meningioma.

Recently, Dong et al. [34] introduced the role of FDG PET/CT as another helpful diagnostic tool in identifying endobronchial metastases.

In conclusion, the current study represents the largest series of consecutive extrapulmonary tumors presenting with endobronchial metastases. Summarizing the key points derived from the current data, it is important to keep in mind that: (1) endobronchial metastases may occur in about $4 \%$ of bronchoscopic examinations performed for a suspected neoplasm; (2) a not insignificant rate of endobronchial metastases ( $5 \%$ in our study) may be the first manifestation of an extra-pulmonary tumor; (3) virtually all kind of solid tumors may occasionally metastasize into the bronchial lumen; (4) up to one third of patients with endobronchial metastasis may be asymptomatic or even present with a radiologic pattern mimicking primary lung cancer.

When endobronchial metastases lead to controversial issues with clinical implications on diagnostic ground, the close collaboration between clinician, radiologist and pathologist is the most critical step in achieving the correct diagnosis, then preventing erroneous therapeutic approaches.

\section{Conflict of interest statement}

No conflicts of interest for all authors.

\section{References}

[1] Jung RC. Pulmonary lesions associated with extrapulmonary malignancies. Semin Respir Med 1988;9:334-442.

[2] Greelish JP, Friedberg JS. Secondary pulmonary malignancy. Surg Clin North Am 2000;80:633-57.

[3] Braman SS, Whitecomb ME. Endobronchial metastasis. Arch Intern Med 1975;135:543-7.

[4] Salud A, Porcel JM, Rovirosa A, Bellmunt J. Endobronchial metastatic disease: analysis of 32 cases. J Surg Oncol 1996;62:249-52.

[5] Froudarakis ME, Bouras D, Siafakas NH. Endoluminal metastases of tracheobronchial tree: is there any way out? Chest 2001;119:679-81.

[6] Sheperd MP. Endobronchial metastatic disease. Thorax 1982;37:362-5.

[7] Kiryu T, Hoshi H, Matsui E, Iwata H, Kokubo M, Shimokawa K, et al. Endotracheal/endobronchial metastases. Chest 2001;119:768-75.

[8] Katsimbri PP, Bamias AT, Froudarakis ME, Peponis IA, Costantopoulos SH, Pavlidis NA. Endobronchial metastases secondary to solid tumors: report of eight cases and review of the literature. Lung Cancer 2000;28:163-70.

[9] Soresen. Endobronchial metastases from extrapulmonary solid tumors. Acta Oncol 2004;43:73-9.

[10] Sebahat A, Eyup SU, Gulperi C, Gulper S, Can S, Oguz K, et al. Endobronchial metastases from extrathoracic malignancies. Clin Exp Metastasis 2005;22:587-91.

[11] Dursun AB, Demirag F, Bayiz H, Sertkaya D. Endobronchial metastases: a clinicopathological analysis. Respirology 2005;10:510-4.

[12] King DS, Castelman B. Bronchial involvement in metastatic pulmonary malignancy. J Thorac Surg 1943;12:305-15.

[13] Baumgartner WA, Mark JBD. Metastatic malignancies from distant sites to the tracheobronchial tree. J Thorac Cardiovasc Surg 1980;79:499-503.

[14] Heitmiller RF, Marasco WJ, Hruban RH, Marsh BR. Endobronchial metastases. J Thorac Cardiovasc Surg 1993;106:537-42.

[15] Higginson JF. A study of excised pulmonary metastatic malignancies. Am J Surg 1995;90:251-2.

[16] Rosenblatt MB, Lisa JR, Trinidad S. Pitfalls in the clinical and histologic diagnosis of bronchogenic carcinoma. Chest 1966;49:396-404.

[17] Debeer RA, Garcia RL, Alexander SC. Endobronchial metastasis from cancer of the breast. Chest 1978;73:94-6.

[18] Albertini RE, Ekberg NL. Endobronchial metastasis in breast cancer. Thorax 1980;35:435-40.

[19] Ettensohn DB, Bennett JM, Hyde RW. Endobronchial metastases from carcinoma of the breast. Med Pediatr Oncol 1985;13:9-13.

[20] Carlin BM, Harrel JH, Olson LK, Moser KM. Endobronchial metastases because of colorectal carcinoma. Chest 1989;96:1110-4.

[21] Rovirosa Casino A, Bellmunt J, Salud A, Vicente P, Maldonado J, Bodi $\mathrm{R}$, et al. Endobronchial metastases in colorectal adenocarcinoma. Tumori 1992;78:270-3.

[22] Jariwalla AG, Seaton A, McCormack RJM, Gibbs A, Campbell IA, Davies BH. Intrabronchial metastases from renal carcinoma with recurrent tumor expectoration. Thorax 1981;36:179-82.

[23] Gerle R, Felson B. Metastatic endobronchial hypernephroma. Chest $1963 ; 44: 225$

[24] Amer E, Guy J, Vaze B. Endobronchial metastasis from renal adenocarcinoma simulating a foreign body. Thorax 1981;36:183-4.

[25] Udelsman R, Roth J, Lees D, Jelenich SE, Pass HI. Endobronchial metastases from soft tissue sarcoma. J Surg Oncol 1986;32:145-9.

[26] Akiba T, Ujiie H, Takasaki N, Ohki T, Kurihara H, Endo Y, et al. Endobronchial metastasis from a primary uterine osteosarcoma in a patient with multiple myeloma: report of a case. Surg Today 1994;24:179-82.

[27] Amin R. Endobronchial metastasis from malignant schwannoma. Br J Radiol $1984 ; 57: 528-30$

[28] Coaker LA, Sobonye RE, Davis JR. Endobronchial metastases from uterine cervical squamous carcinoma. Arch Pathol Lab Med 1984;108:269-71.

[29] Scherz H, Schmidt JD. Endobronchial metastases from prostate carcinoma. Prostate 1986;8:319-24. 
[30] Kao H, Yu CP, Tzao C, Lin WC, Hsu HH, Chen CY. An unusual case of thymic carcinoma with endobronchial metastases manifesting as centrilobular opacities. J Thorac Imaging 2006;21:238-40.

[31] Kreisman H, Wolkove N, Finkelstein HS, Cohen C, Margolese R, Frank H. Breast cancer and thoracic metastases: review of 119 patients. Thorax 1983;38: 175-9.

[32] Lee SH, Jung JY, Kim DH, Lee SK, Kim SY, Kim EY, et al. Endobronchial metastases from extrathoracic malignancy. Yonsei Med J 2013;54: 403-9.
[33] Kim JH, Min D, Song SH, Lee JH, Jeong HC, Kim EK. Endobronchial metastases from extrathoracic malignancies: recent 10 years' experience in a single University Hospital. Tuberc Respir Dis 2013;74:169-76.

[34] Dong A, Zhao T, Wang Y, Zuo C. FDG PET/CT in endobronchial metastases from extrathoracic malignancies. Clin Nucl Med 2013 [Epub ahead of print].

[35] Whitesell PL, Peters SG. Pulmonary manifestations of extrathoracic malignan lesions. Mayo Clin Proc 1993;68:483-91.

[36] Suster S, Morac CA. Unusual manifestations of metastatic tumors of the lungs. Semin Diagn Pathol 1995;12:193-206. 